\title{
Tolvaptan is effective in treating patients with refractory ascites due to cirrhosis
}

\author{
TOSHIYUKI TAHARA ${ }^{1}$, KIYOTO MORI ${ }^{1}$, MARI MOCHIZUKI ${ }^{1}$, RYOKO ISHIYAMA ${ }^{1}$, \\ MARIN NODA $^{1}$, HITOMI HOSHI ${ }^{1}$, ALAN KAWARAI LEFOR ${ }^{2}$ and SATOSHI SHINOZAKI ${ }^{3,4}$ \\ ${ }^{1}$ Department of Gastroenterology, Saiseikai Utsunomiya Hospital, Utsunomiya, Tochigi 329-0974; \\ ${ }^{2}$ Department of Surgery, Jichi Medical University, Shimotsuke, Tochigi 329-0498; ${ }^{3}$ Shinozaki Medical Clinic, \\ Utsunomiya, Tochigi 321-3223; ${ }^{4}$ Division of Gastroenterology, Department of Medicine, \\ Jichi Medical University, Shimotsuke, Tochigi 329-0498, Japan
}

Received August 21, 2017; Accepted October 17, 2017

DOI: 10.3892/br.2017.1005

\begin{abstract}
The treatment of refractory ascites due to cirrhosis is a clinical challenge for hepatologists. Tolvaptan, a novel aquaporin modulator, was made available in Japan in 2013 for the treatment of patients with refractory ascites due to cirrhosis. Despite the potential of this drug, few reports are available regarding its clinical use. The aim of the present study was to clarify the efficacy of tolvaptan in patients with refractory ascites due to cirrhosis and to review the clinical outcomes of treatment. Medical records were retrospectively reviewed for 65 patients with refractory ascites due to cirrhosis who were treated daily with $7.5 \mathrm{mg}$ tolvaptan. The median follow-up time, defined as the period between starting tolvaptan and the last clinic visit or date of mortality, was 175 days (interquartile range 56-406). After one week of tolvaptan treatment, the mean weight reduction was $3.4 \mathrm{~kg}$, with a response rate of $69 \%$ (45/65). Subsequently, factors associated with the response to tolvaptan were analyzed. On univariate analysis, maintaining serum sodium (Na) $\geq 140 \mathrm{mEq} / \mathrm{l}$ and an estimated glomerular filtration rate $(\mathrm{eGFR}) \geq 55 \mathrm{ml} / \mathrm{min}$ were significant predictors of response $(\mathrm{P}<0.05)$. On multivariate analysis, hepatitis $\mathrm{C}$ virus etiology, maintaining serum $\mathrm{Na} \geq 140 \mathrm{mEq} / \mathrm{l}$ and an eGFR $\geq 55 \mathrm{ml} / \mathrm{min}$ were significant predictors of response $(\mathrm{P}<0.05)$. Factors associated with survival were also analyzed using the Cox proportional hazard model. On multivariate analysis, responsiveness to tolvaptan was a predictor of long-term survival $(\mathrm{P}=0.002)$, and hyperbilirubinemia was associated
\end{abstract}

Correspondence to: Dr Satoshi Shinozaki, Shinozaki Medical Clinic, 6-1-13 Kiyoharadai, Utsunomiya, Tochigi 321-3223, Japan

E-mail: shinozaki-s@aqua.ocn.ne.jp

Abbreviations: eGFR, estimated glomerular filtration rate; HCC, hepatocellular carcinoma; HCV, hepatitis C virus; MELD, Model for End-Stage Liver Disease

Key words: liver cirrhosis, ascites, diuretics, patient outcome assessment, hyponatremia, glomerular filtration rate with short-term survival $(\mathrm{P}=0.028)$. Additionally, KaplanMeier analysis with a log-rank test indicated longer survival times in tolvaptan responders than non-responders $(\mathrm{P}=0.011)$. In conclusion, tolvaptan was effective in treating patients with refractory ascites due to cirrhosis. In particular, tolvaptan treatment was highly effective for patients with hepatitis $\mathrm{C}$ virus etiology and normal serum $\mathrm{Na}$ and renal function. Furthermore, response to tolvaptan was associated with longer survival time while hyperbilirubinemia was associated with shorter survival time.

\section{Introduction}

The treatment of cirrhotic patients with refractory ascites is a clinical challenge for hepatologists. Refractory ascites is defined as ascites that cannot be resolved using standard doses of diuretics, namely spironolactone, loop and thiazide diuretics, among others (1). Patients with advanced cirrhosis have a decreased estimated glomerular filtration rate (eGFR) due to diminished circulating volume (2). This is partially due to increased reabsorption in the proximal convoluted tubule, resulting in a poor current in the distal convoluted tubule. Consequently, the effect of vasopressin on the collecting duct is blunted, resulting in sodium and water retention (2). Additionally, hypoalbuminemia due to cirrhosis induces intravascular dehydration through decreased colloid osmotic pressure. This depleted circulation volume may decrease the effect of standard diuretics (2).

Tolvaptan, a novel aquaporin modulator, was made available in Japan in 2013 for the treatment of patients with refractory ascites due to cirrhosis (3). Tolvaptan enhances renal function by inhibiting water reabsorption in the collecting duct, namely by preventing the binding of vasopressin to $\mathrm{V} 2$ receptors and thus suppressing the expression of aquaporin-2 (3). This therapeutic mechanism has the potential to improve cirrhosisinduced ascites, and may decrease the need for paracentesis, cell-free and concentrated ascites reinfusion therapy, and transjugular intrahepatic portosystemic shunt placement. A phase III trial of tolvaptan in Japan observed a marked reduction in fluid retention in patients with refractory ascites due to cirrhosis (4). Additionally, tolvaptan may improve refractory 
ascites even in patients with hypoalbuminemia (5). However, despite the therapeutic potential of tolvaptan, few reports are available on its clinical use, particularly regarding long-term outcomes. Thus, the present study aimed to clarify the shortterm efficacy and review the long-term clinical outcomes of tolvaptan in patients with refractory ascites due to cirrhosis.

\section{Patients and methods}

Study design. From June 2013 to April 2017, 89 patients with refractory ascites due to cirrhosis were treated with tolvaptan at Saiseikai Utsunomiya Hospital (Utsunomiya, Japan). The patient medical records were retrospectively reviewed and data were collected regarding the following parameters: Age, gender, weight, body mass index, smoking habits, etiology of cirrhosis, Child-Pugh classification (6), Model for End-Stage Liver Disease (MELD) score (7), the presence of comorbidities, varices, viable hepatocellular carcinoma (HCC) and/or pleural effusion, history of hepatic encephalopathy, previous doses of standard diuretics, laboratory findings and long-term follow-up data. The presence of varices, HCC and pleural effusion was determined by esophagogastroduodenoscopy, computed tomography/ultrasound and chest X-ray. All patients were hospitalized for the control of refractory ascites that had not been controlled by standard diuretics (furosemide $\geq 20 \mathrm{mg} /$ day and/or spironolactone $\geq 25 \mathrm{mg} /$ day). The starting dose of tolvaptan was $7.5 \mathrm{mg}$ daily for all patients. During hospitalization, sodium $(\mathrm{Na})$ chloride intake was limited to $6 \mathrm{~g} /$ day while fluid intake was not limited. At present, there is no formal criteria to define a 'responder' to diuretic treatment in patients with refractory ascites due to cirrhosis. Therefore, a "responder" was defined in the present study by $\geq 2 \mathrm{~kg}$ weight reduction within seven days of starting tolvaptan. The follow-up time was defined as the period between starting tolvaptan and the last clinic visit or date of mortality. To obtain homogeneous data, 24 of the 89 patients were excluded for the following reasons: Failure to measure weight $(n=11)$, half-dose $(3.75 \mathrm{mg})$ tolvaptan intake $(\mathrm{n}=6)$, ascites drained by paracentesis within seven days of starting tolvaptan $(n=4)$ and warfarin use $(n=3)$. The remaining 65 patients were analyzed as the final cohort. The study was approved by the Institutional Review Board of Saiseikai Utsunomiya Hospital (Utsunomiya, Japan) prior to data collection.

Statistical analysis. Cut-off values were determined using a receiver operating characteristic curve to convert continuous data into categorical data. To identify predictive factors associated with response to tolvaptan, univariate and multivariate logistic regression analyses were performed. For multivariate analysis, factors were selected by the stepwise forward selection method. To assess long-term follow-up data, risk factors associated with survival were analyzed with the Cox proportional hazards model. The Kaplan-Meier method with a log-rank test was used to compare the survival curves of tolvaptan responders and non-responders, and Child-Pugh scores prior to and at six months of tolvaptan therapy were compared using a Wilcoxon rank sum test. Statistical analysis was performed using StatFlex 6.0 software (Artech Co., Ltd., Osaka, Japan) and differences were considered significant when $\mathrm{P}<0.05$.
Table I. Baseline characteristics and patient outcomes.

\begin{tabular}{|c|c|}
\hline Variable & Total, $\mathrm{n}=65$ \\
\hline Age, years, mean \pm SD & $71.5 \pm 9.1$ \\
\hline Gender, male, n (\%) & $41(63)$ \\
\hline Weight, kg, mean \pm SD & $62.4 \pm 15.4$ \\
\hline Body mass index, mean \pm SD & $24.5 \pm 4.6$ \\
\hline Current Smoker, n (\%) & $9(14 \%)$ \\
\hline \multicolumn{2}{|l|}{ Etiology, n (\%) } \\
\hline $\mathrm{HCV}$ & $40(62)$ \\
\hline Alcohol & $12(18)$ \\
\hline AIH & $4(6)$ \\
\hline $\mathrm{PBC}$ & $3(5)$ \\
\hline NASH & $1(1)$ \\
\hline Unknown & $5(8)$ \\
\hline \multicolumn{2}{|l|}{ Child-Pugh classification, class } \\
\hline $\mathrm{B}$ & $28(43)$ \\
\hline $\mathrm{C}$ & $37(57)$ \\
\hline MELD score, mean \pm SD & $12.4 \pm 4.8$ \\
\hline \multicolumn{2}{|l|}{ Comorbidities, n (\%) } \\
\hline Diabetes mellitus & $24(37)$ \\
\hline Hypertension $^{\mathrm{a}}$ & $10(15)$ \\
\hline Heart failure & $3(5)$ \\
\hline \multicolumn{2}{|l|}{ Liver related complications, n (\%) } \\
\hline Varices & $49(75)$ \\
\hline Viable hepatocellular carcinoma & $28(43)$ \\
\hline History hepatic encephalopathy & $23(35)$ \\
\hline Current pleural effusion & $6(9)$ \\
\hline \multicolumn{2}{|l|}{ Diuretics, n (\%); mg/day, mean \pm SD } \\
\hline Furosemide & $54(83) ; 43.5 \pm 20.8$ \\
\hline Spironolactone & $54(83) ; 48.6 \pm 27.9$ \\
\hline Azosemide & $12(18) ; 46.7 \pm 14.2$ \\
\hline \multicolumn{2}{|l|}{ Biochemistry } \\
\hline Platelet, x $10,000 / \mu 1)$ & $12.4 \pm 8.5$ \\
\hline Albumin, $\mathrm{g} / \mathrm{dl}$ & $2.3 \pm 0.5$ \\
\hline Total bilirubin, mg/dl & $2.0 \pm 1.9$ \\
\hline Serum Na, mEq/l & $137.0 \pm 4.7$ \\
\hline Serum K, mEq/l & $3.9 \pm 0.5$ \\
\hline $\mathrm{eGFR}, \mathrm{ml} / \mathrm{min}$ & $55.1 \pm 25.6$ \\
\hline PT-INR & $1.3 \pm 0.3$ \\
\hline $\begin{array}{l}\text { Weight reduction over initial } \\
\text { seven days, } \mathrm{kg} \text {, mean } \pm \mathrm{SD}\end{array}$ & $3.4 \pm 2.9$ \\
\hline Tolvaptan responder, n (\%) & $45(69)$ \\
\hline $\begin{array}{l}\text { Length of first hospital stay, } \\
\text { days, median (IQR) }\end{array}$ & $14(9-24)$ \\
\hline Follow-up period, days, median (IQR) & $175(56-406)$ \\
\hline
\end{tabular}

${ }^{a}$ Hypertension as defined by the Japanese Hypertension Society Guidelines (20). Comorbidities were treated with appropriate medications. SD, standard deviation; $\mathrm{HCV}$, hepatitis $\mathrm{C}$ virus; $\mathrm{AIH}$, autoimmune hepatitis; $\mathrm{PBC}$, primary biliary cirrhosis; NASH, nonalcoholic steatohepatitis; MELD, model for end-stage liver disease; eGFR, estimated glomerular filtration rate; PT-INR: prothrombin time-international normalized ratio; IQR, interquartile range. 
Table II. Factors associated with tolvaptan response assessed by logistic regression analysis.

\begin{tabular}{|c|c|c|c|c|c|c|}
\hline \multirow[b]{2}{*}{ Factor } & \multicolumn{3}{|c|}{ Univariate analysis } & \multicolumn{3}{|c|}{ Multivariate analysis } \\
\hline & OR & $95 \% \mathrm{CI}$ & P-value & OR & $95 \% \mathrm{CI}$ & P-value \\
\hline Age, $\geq 75$ years & 2.041 & $0.665-6.266$ & 0.212 & - & - & - \\
\hline Male gender & 0.886 & $0.295-2.661$ & 0.830 & - & - & - \\
\hline Hepatitis $\mathrm{C}$ virus infection & 2.706 & $0.915-7.998$ & 0.071 & 4.998 & $1.214-20.576$ & 0.025 \\
\hline Viable hepatocellular carcinoma & 0.893 & $0.309-2.580$ & 0.834 & - & - & - \\
\hline Platelet, $\geq 90,000 / \mu 1$ & 1.647 & $0.568-4.771$ & 0.357 & - & - & - \\
\hline Serum albumin, $\geq 2.3 \mathrm{mg} / \mathrm{dl}$ & 0.583 & $0.200-1.699$ & 0.323 & - & - & - \\
\hline Total bilirubin, $\geq 1.7 \mathrm{mg} / \mathrm{dl}$ & 2.231 & $0.727-6.846$ & 0.160 & - & - & - \\
\hline Serum $\mathrm{Na}, \geq 140 \mathrm{mEq} / \mathrm{l}$ & 5.420 & $1.391-21.106$ & 0.014 & 8.291 & $1.641-41.870$ & 0.010 \\
\hline $\mathrm{eGFR}, \geq 55 \mathrm{ml} / \mathrm{min}$ & 3.826 & $1.105-13.245$ & 0.034 & 6.890 & $1.442-32.921$ & 0.015 \\
\hline PT-INR, $\geq 1.4$ & 2.206 & $0.629-7.735$ & 0.216 & - & - & - \\
\hline History of encephalopathy & 1.416 & $0.457-4.387$ & 0.545 & - & - & - \\
\hline
\end{tabular}

For multivariate analysis factors were selected by the stepwise forward selection method. eGFR, estimated glomerular filtration rate; PT-INR, prothrombin time-international normalized ratio; OR, odds ratio; CI, confidence interval.

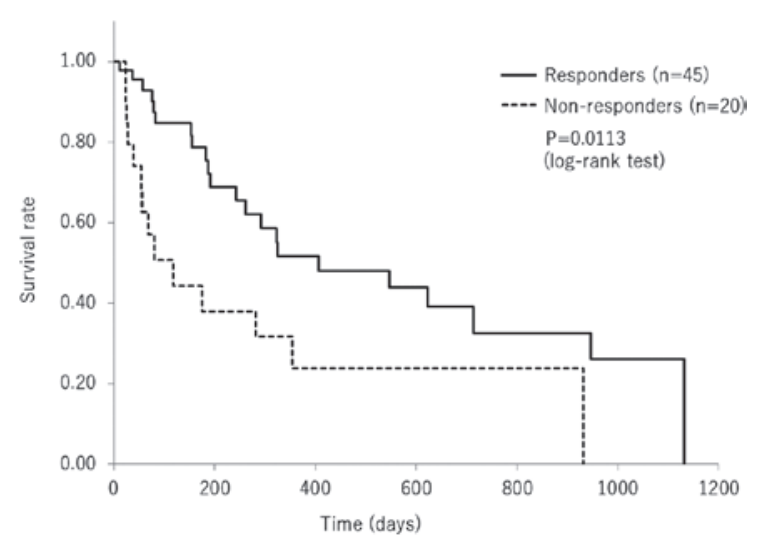

Figure 1. Kaplan-Meier survival curves for tolvaptan responders and non-responders.

\section{Results}

Baseline characteristics and short-term outcomes. The mean age of the patients was $71.5 \pm 9.1$ years, and $62 \%(40 / 65)$ exhibited hepatitis C virus (HCV) etiology, while 57\% (37/65) were classified as class $\mathrm{C}$ of the Child-Pugh classification (Table I). All 40 patients with HCV etiology exhibited a positive HCV-RNA tests. All patients received furosemide ( $\geq 20 \mathrm{mg} /$ day) and/or spironolactone ( $\geq 25 \mathrm{mg} /$ day) prior to starting tolvaptan therapy. After one week of tolvaptan treatment, the mean weight reduction was $3.4 \pm 2.9 \mathrm{~kg}$, with a $69 \%$ (45/65) response rate to tolvaptan (Table I). No adverse events occurred during the hospital stay.

Factors associated with tolvaptan responders. Factors associated with response to tolvaptan were analyzed (Table II). On univariate analysis, maintaining serum $\mathrm{Na} \geq 140 \mathrm{mEq} / \mathrm{l}$ and eGFR $\geq 55 \mathrm{ml} / \mathrm{min}$ were significantly associated with response to tolvaptan $(\mathrm{P}<0.05)$. On multivariate analysis, HCV etiology, maintaining serum $\mathrm{Na} \geq 140 \mathrm{mEq} / 1$ and $\mathrm{eGFR} \geq 55 \mathrm{ml} / \mathrm{min}$ were significantly associated with response $(\mathrm{P}<0.05)$.

Long-term outcomes. The long-term outcomes of tolvaptan therapy were evaluated. The median follow-up period was 175 days (interquartile range 56-406). During the follow-up period, a total of 36 patients succumbed, due to hepatic failure $(n=20)$, hepatocellular carcinoma $(n=13)$, gastrointestinal bleeding $(n=2)$ and heart failure $(n=1)$. Risk factors associated with survival were analyzed using the Cox proportional hazards model. On univariate analysis, tolvaptan responsiveness was a predictor of long-term survival $(\mathrm{P}=0.012)$, while viable HCC was a predictor of short-term survival ( $\mathrm{P}=0.044$; Table III). On multivariate analysis, responsiveness to tolvaptan remained a predictor of long-term survival $(\mathrm{P}=0.002)$, while hyperbilirubinemia was a predictor of short-term survival $(\mathrm{P}=0.028$; Table III). Kaplan-Meier analysis of survival with a log-rank test indicated a significantly longer overall survival time of responders compared with non-responders $(\mathrm{P}=0.011$; Fig. 1).

After six months of tolvaptan therapy, 19 patients had succumbed and 15 were lost to follow-up. The remaining 31 patients were followed for more than six months. Child-Pugh scores of the patients were assessed prior to and six months after starting tolvaptan therapy to evaluate hepatic function. For the 31 patients followed for six months and longer, the Child-Pugh scores were significantly decreased at six months $(8.5 \pm 2.1)$ compared with those prior to starting tolvaptan $(9.7 \pm 1.2 ; \mathrm{P}<0.001)$.

\section{Discussion}

The present retrospective cohort study demonstrated that tolvaptan was an effective treatment for cirrhotic patients with refractory ascites. Notably, HCV etiology and maintaining serum $\mathrm{Na} \geq 140 \mathrm{mEq} / 1$ and renal function $\geq 55 \mathrm{ml} / \mathrm{min}$ were significantly associated with response to tolvaptan on 
Table III. Risk factors associated with survival analyzed by the Cox proportional hazards model.

\begin{tabular}{|c|c|c|c|c|c|c|}
\hline \multirow[b]{2}{*}{ Factor } & \multicolumn{3}{|c|}{ Univariate analysis } & \multicolumn{3}{|c|}{ Multivariate analysis } \\
\hline & HR & $95 \% \mathrm{CI}$ & P-value & HR & $95 \% \mathrm{CI}$ & P-value \\
\hline Responder to tolvaptan & 0.418 & $0.210-0.830$ & 0.012 & 0.269 & $0.113-0.638$ & 0.002 \\
\hline Age, $\geq 75$ years & 1.410 & $0.709-2.804$ & 0.326 & - & - & - \\
\hline Male gender & 1.253 & $0.632-2.484$ & 0.517 & 1.609 & $0.726-3.565$ & 0.241 \\
\hline Hepatitis $\mathrm{C}$ virus infection & 1.953 & $0.883-4.323$ & 0.098 & 2.170 & $0.906-5.198$ & 0.082 \\
\hline Viable hepatocellular carcinoma & 2.008 & $1.016-3.969$ & 0.044 & 1.336 & $0.581-3.072$ & 0.495 \\
\hline Platelet, $\geq 90,000 / \mu 1$ & 0.789 & $0.399-1.559$ & 0.495 & - & - & - \\
\hline Serum albumin, $\geq 2.3 \mathrm{mg} / \mathrm{dl}$ & 0.976 & $0.501-1.900$ & 0.944 & - & - & - \\
\hline Total bilirubin, $\geq 1.7$ mg/dl & 1.425 & $0.718-2.825$ & 0.310 & 2.447 & $1.098-5.455$ & 0.028 \\
\hline Serum Na, $\geq 140 \mathrm{mEq} / \mathrm{l}$ & 0.648 & $0.326-1.289$ & 0.216 & - & - & - \\
\hline Estimated GFR, $\geq 55 \mathrm{ml} / \mathrm{min}$ & 0.613 & $0.302-1.244$ & 0.175 & - & - & - \\
\hline PT-INR, $\geq 1.4$ & 1.210 & $0.563-2.602$ & 0.624 & - & - & - \\
\hline History of encephalopathy & 1.212 & $0.605-2.430$ & 0.586 & - & - & - \\
\hline
\end{tabular}

For multivariate analysis factors were selected by the stepwise forward selection method. eGFR, estimated glomerular filtration rate; PT-INR, prothrombin time-international normalized ratio; HR, hazard ratio; $\mathrm{CI}$, confidence interval.

multivariate analysis. Additionally, response to tolvaptan was associated with improved long-term outcomes in cirrhotic patients with refractory ascites.

The short-term effects of tolvaptan in patients with refractory ascites due to cirrhosis have been reported (8-13). As the starting doses in previous studies were $3.75 \mathrm{mg}$ with dose escalation up to $7.5 \mathrm{mg}$ within one week, tolvaptan doses in these studies were heterogeneous. The dose of tolvaptan was consistent at $7.5 \mathrm{mg}$ per day in the current study. In prior studies with a starting dose of tolvaptan of $3.75 \mathrm{mg}$ daily, weight reduction within one week ranged from $1.5-3.8 \mathrm{~kg}(8-11,13)$. The mean weight reduction at one week was $3.4 \pm 2.9 \mathrm{~kg}$ in the present study. Thus, the lower dose of tolvaptan used in prior studies $(3.75 \mathrm{mg}$ ) may be as effective as a higher dose $(7.5 \mathrm{mg})$. However, the optimal starting dose of tolvaptan is yet to be determined in future studies.

In the current study, HCV-related cirrhosis was a positive predictor for response to tolvaptan, as reported by Ohki et al previously (11), though the reason for this association remains unclear. A possible explanation may be that alcoholrelated cirrhosis was the etiology for $\sim$ half of the patients with non-HCV-related cirrhosis in the current study. Patients with alcohol-related cirrhosis may not be as strictly followed compared with HCV-infected individuals, and may have a delay in treatment with tolvaptan. Additionally, patients with alcohol-related cirrhosis possibly have greater intravascular dehydration than patients with HCV-related cirrhosis, which may affect their response.

In the present study, patients with normal renal function exhibited an adequate response to tolvaptan. It was also identified that serum $\mathrm{Na}(>140 \mathrm{mEq} / \mathrm{l})$ was a positive predictor of response to tolvaptan. Umemura et al reported that serum $\mathrm{Na}$ $<139 \mathrm{mEq} / \mathrm{l}$ was significantly associated with mortality in patients with cirrhosis (14). Additionally, hyponatremia has been documented to be an independent predictor for poor prognosis in patients with cirrhosis (7), and is observed in half of cirrhotic individuals (15). Recent studies have also reported that low serum creatinine is a predictor of response to tolvaptan in the short-term in univariate analyses $(8,9)$. Deterioration of renal function in patients with cirrhosis directly influences their prognosis (7). In contrast to standard diuretics, which cause hyponatremia and glomerular damage with continuous and high dose administration, no hyponatremia or glomerular damage has been observed with tolvaptan treatment (8). Additionally, unlike standard diuretics, tolvaptan is not affected by serum albumin level (5). Tolvaptan may also resolve hyponatremia, potentially resulting in improved survival (1,7). Although administration of albumin may be a viable treatment option in patients with refractory ascites, albumin is a high-cost medical biological product. Therefore, the use of tolvaptan may be appropriate in the early stages of cirrhosis prior to high-dose administration of standard diuretics.

There are few reports of the long-term outcomes of tolvaptan therapy in cirrhotic patients with refractory ascites. Response to tolvaptan was associated with improved survival in the current study. Previous studies have reported similar results from univariate analyses $(8,9)$. As aforementioned, renal function and serum $\mathrm{Na}$ level may be considered as major indicators of tolvaptan responsiveness, particularly as these are established prognostic factors in patients with cirrhosis (7). To exclude confounding factors, the present study used multivariate analysis and identified response to tolvaptan as a positive predictor for survival. A recent Japanese study reported similar results based on multivariate analysis (13). It is probable that patients who exhibit alleviated ascites are in less advanced disease stages, and therefore have better prognosis. Regardless, a preserved serum sodium and high eGFR, which may indicate preserved cardiocirculatory function, may be potential predictors of tolvaptan responsiveness. Furthermore, 
total serum bilirubin level is an established negative prognostic marker in patients with cirrhosis and is included in the MELD score (16).

Tolvaptan has been used for the treatment of patients with heart failure worldwide (17). The Japanese health insurance system began reimbursement for tolvaptan therapy in cirrhotic patients with refractory ascites in 2013, as the first country globally (3). Prior to the reimbursement of tolvaptan therapy in Japan, dose escalation of spironolactone, loop and thiazide diuretics had been the first-choice strategy for the medical management of patients with refractory ascites, despite its associations with glomerular damage and hyponatremia (3). Although abdominal paracentesis with drainage, cell-free and concentrated ascites reinfusion therapy and trans-jugular intrahepatic portosystemic shunt placement are occasionally used, results suggest that these procedures do not improve overall prognosis (18-20). Tolvaptan may delay the need for invasive procedures and readmission for patients with refractory ascites.

The present study had some limitations. Firstly, it was a single center, retrospective study. Secondly, patients treated without tolvaptan were not included as a control group. Thirdly, the study defined a 'responder' by $\geq 2 \mathrm{~kg}$ weight reduction. As body weight reduction may be caused various factors including gastroenteritis, other parameters such as abdominal circumference should have been measured. A future prospective study with a large number of patients and longer follow-up is necessary to confirm the current preliminary results.

In conclusion, tolvaptan was effective for the treatment of patients with refractory ascites due to cirrhosis. Tolvaptan was more effective in patients with $\mathrm{HCV}$ etiology, a maintained serum $\mathrm{Na} \geq 140 \mathrm{mEq} / \mathrm{l}$ and renal function $(\mathrm{eGFR}) \geq 55 \mathrm{ml} / \mathrm{min}$. Additionally, response to tolvaptan was a positive independent predictor of survival, while hyperbilirubinemia was a negative predictor of survival. Therefore, tolvaptan therapy may improve the long-term outcomes of patients with cirrhosis through its potential therapeutic effect on refractory ascites.

\section{References}

1. Dahl E, Gluud LL, Kimer N and Krag A: Meta-analysis: The safety and efficacy of vaptans (tolvaptan, satavaptan and lixivaptan) in cirrhosis with ascites or hyponatraemia. Aliment Pharmacol Ther 36: 619-626, 2012.

2. Kawaratani H, Fukui $\mathrm{H}$ and Yoshiji $\mathrm{H}$ : Treatment for cirrhotic ascites. Hepatol Res: Jul 1, 2016 (Epub ahead of print).

3. Kurosaki M and Izumi N: Tolvaptan for the Treatment of Refractory Ascites. Intern Med 55: 2909-2910, 2016.

4. Sakaida I, Kawazoe S, Kajimura K, Saito T, Okuse C, TakaguchiK, Okada $\mathrm{M}$ and Okita K; ASCITES-DOUBLEBLIND Study Group: Tolvaptan for improvement of hepatic edema: A phase 3 , multicenter, randomized, double-blind, placebo-controlled trial. Hepatol Res 44: 73-82, 2014.

5. Sakaida I, Nakajima K, Okita K, Hori M, Izumi T, Sakurai M, Shibasaki Y, Tachikawa S, Tsubouchi H, Oka H, et al: Can serum albumin level affect the pharmacological action of tolvaptan in patients with liver cirrhosis? A post hoc analysis of previous clinical trials in Japan. J Gastroenterol 50: 1047-1053, 2015.
6. Pugh RN, Murray-Lyon IM, Dawson JL, Pietroni MC and Williams R: Transection of the oesophagus for bleeding oesophageal varices. Br J Surg 60: 646-649, 1973.

7. Londoño MC, Cárdenas A, Guevara M, Quintó L, de Las Heras D, Navasa M, Rimola A, Garcia-Valdecasas JC, Arroyo V and Ginès P: MELD score and serum sodium in the prediction of survival of patients with cirrhosis awaiting liver transplantation. Gut 56: 1283-1290, 2007

8. Iwamoto T, Maeda M, Hisanaga T, Saeki I, Fujisawa K, Matsumoto T, Hidaka I, Ishikawa T, Takami T and Sakaida I: Predictors of the Effect of Tolvaptan on the Prognosis of Cirrhosis. Intern Med 55: 2911-2916, 2016.

9. Kogiso T, Yamamoto K, Kobayashi M, Ikarashi Y, Kodama K, Taniai M, Torii N, Hashimoto E and Tokushige K: Response to tolvaptan and its effect on prognosis in cirrhotic patients with ascites. Hepatol Res 47: 835-844, 2017.

10. Akiyama S, Ikeda K, Sezaki H, Fukushima T, Sorin Y, Kawamura Y, Saitoh S, Hosaka T, Akuta N, Kobayashi M: Therapeutic effects of short- and intermediate-term tolvaptan administration for refractory ascites in patients with advanced liver cirrhosis. Hepatol Res 45: 1062-1070, 2015.

11. Ohki T, Sato K, Yamada T, Yamagami M, Ito D, Kawanishi K, Kojima K, Seki M, Toda N and Tagawa K: Efficacy of tolvaptan in patients with refractory ascites in a clinical setting. World J Hepatol 7: 1685-1693, 2015.

12. Komiyama Y, Kurosaki M, Nakanishi H, Takahashi Y, Itakura J, Yasui Y, Tamaki N, Takada H, Higuchi M, Gotou T, et al: Prediction of diuretic response to tolvaptan by a simple, readily available spot urine Na/K ratio. PLoS One 12: e0174649, 2017.

13. Yamada T, Ohki T, Hayata Y, Karasawa Y, Kawamura S, Ito D, Kojima K, Seki M, Toda N and Tagawa K: Potential Effectiveness of Tolvaptan to Improve Ascites Unresponsive to Standard Diuretics and Overall Survival in Patients with Decompensated Liver Cirrhosis. Clin Drug Investig 36: 829-835, 2016.

14. Umemura T, Shibata S, Sekiguchi T, Kitabatake H, Nozawa Y, Okuhara S, Kimura T, Morita S, Komatsu M, Matsumoto A and Tanaka E: Serum sodium concentration is associated with increased risk of mortality in patients with compensated liver cirrhosis. Hepatol Res 45: 739-744, 2015.

15. Angeli P, Wong F, Watson H and Ginès P; CAPPS Investigators: Hyponatremia in cirrhosis: Results of a patient population survey. Hepatology 44: 1535-1542, 2006.

16. Kamath PS, Wiesner RH, Malinchoc M, Kremers W, Therneau TM, Kosberg CL, D'Amico G, Dickson ER and Kim WR: A model to predict survival in patients with end-stage liver disease. Hepatology 33: 464-470, 2001.

17. Imamura T, Kinugawa K, Shiga T, Kato N, Muraoka H, Minatsuki S, Inaba T, Maki H, Hatano M, Yao A, et al: Novel criteria of urine osmolality effectively predict response to tolvaptan in decompensated heart failure patients- -association between non-responders and chronic kidney disease. Circ J 77: 397-404, 2013.

18. Tan HK, James PD, Sniderman KW and Wong F: Long-term clinical outcome of patients with cirrhosis and refractory ascites treated with transjugular intrahepatic portosystemic shunt insertion. J Gastroenterol Hepatol 30: 389-395, 2015.

19. Matsusaki K, Ohta K, Yoshizawa A and Gyoda Y; Japanese CART Study Group: Novel cell-free and concentrated ascites reinfusion therapy (KM-CART) for refractory ascites associated with cancerous peritonitis: Its effect and future perspectives. Int J Clin Oncol 16: 395-400, 2011.

20. Kario K: Key Points of the Japanese Society of Hypertension Guidelines for the Management of Hypertension in 2014. Pulse Basel 3: 35-47, 2015. 